\title{
Neophobias and conditioned taste aversions in rats following exposure to novel flavors
}

\author{
RALPH R. MILLER and ARNOLD D. HOLZMAN \\ State University of New York, Binghamton, New York 13901
}

\begin{abstract}
Rats were successively exposed to three solutions with distinctively different flavors and then tested for both neophobia and propensity to form conditioned taste aversions to a fourth distinctively flavored solution. All permutations between the four solutions (salty, bitter, sweet, and sour) were examined. The prior exposures resulted in attenuation of neophobia to novel salty and sour solutions, but not to equally novel bitter or sweet solutions. These effects were found to depend upon the diversity of the prior ingestive events rather than upon either a single specific flavor experience or a summation of the reductions in generalized neophobia accrued by each substance separately; both of the latter findings are inconsistent with stimulus generalization being responsible for the observed attenuation of neophobia to salty and sour solutions following exposure to diverse different solutions. A further test of generalization between the salty and bitter solutions, consisting of associating one flavor with poison and extinguishing the avoidance response in half the animals prior to testing for generalization of conditioned taste aversion to the other flavor, also proved negative. Although these effects of exposure to flavors distinctly different from the test solution may be dependent upon solution concentrations, further research found that the same pretest exposures and same test concentrations failed to inhibit formation of conditioned taste aversions. A demonstration of "latent inhibition" attested to the sensitivity of our procedure to potential interference with acquisition of conditioned taste aversions. The results are considered in light of the relationship between neophobia and conditioned tasted aversions, the differential biological relevancy of specific tastes, and abstraction as a cognitive capability of rats. The possibility is raised that the defense against toxins is not the primary function of neophobia.
\end{abstract}

Barnett and Cowan (1976) argue that neophobia is prominent among rats owing to their varied diets and perhaps augmented by the selective pressure of man's attempt to control them with poison. They contend that neophobia may have evolved in rats specifically because of the high likelihood of toxins' occurring in novel, untried substances. This view cannot readily explain the finding that exposure to novelty per se, that is, a varied diet, decreases neophobia to all novel substances, including items that are quite dissimilar to any component of the varied diet (e.g., Braveman \& Jarvis, 1978). Moreover, Barnett and Cowan (1976), among others, have suggested, on the theoretical grounds of common function, that neophobia and conditioned taste aversion (CTA) are merely different points on a common dimension of aversive-

The research in this paper was funded by National Science Foundation Grant BMS75-03383 and NIMH Grant MH-33881. R.R.M. was supported by Research Scientist Development Award K2-MH00061. The authors are indebted to Professor O. L. Zangwill for the hospitality of the Cambridge University Laboratory for Experimental Psychology, Alvin M. Berk for his assistance in the collection of part of the data, Nancy A. Marlin and Anthony Sclafani for their critical reading of an early draft of the manuscript, and Joan L. Wessely for her assistance in preparing the manuscript. Reprint requests should be sent to Ralph R. Miller, Department of Psychology, SUNY-Binghamton, Binghamton, New York 13901. ness. Support for this view is often seen in the decreases in both neophobia and the potential to form CTAs (latent inhibition) that follow uneventful ingestion of a substance. However, several recent studies have reported substantial decrements in neophobia to a specific substance without corresponding changes in the potential to form CTAs (e.g., Braveman \& Jarvis, 1978). If the reduction of neophobia with diverse taste experience and the dissociation of neophobia from CTA propensity prove to be reliable and to generalize across different test flavors, then theory must yield to data, and Barnett and Cowan's functional view of neophobia must be revised. The primary motivation for the present experiments was to examine the reliability and generality of these two effects. Specifically, we investigated the effect of prior novel taste experiences upon neophobia and upon propensity to form CTAs to dissimilar flavors.

The effects of prior gustatory experience on neophobia and the acquisition of CTA have received considerable attention. Consistent with the definition of neophobia, prior exposure to a distinctive test substance augments later acceptance of that substance (e.g., Domjan, 1976; Rzoska, 1953). And, paralleling the "latent inhibition" phenomenon of Lubow and Moore (1.959), pretraining exposure to the test substance retards CTA acquisition (e.g., 
Domjan, 1972; Kalat \& Rozin, 1973; McLaurin, Farley, \& Scarborough, 1963; Siegel, 1974). Moreover, as stimuli can never be exactly duplicated, both of these effects must be subject to generalization gradients around the stimulus characteristics of the taste to which the animal receives prior exposure.

Recently, several investigators have reported studies of neophobia and CTA in which the subjects received prior exposure to palatable substances that differed substantially from the test solution. The apparent differences between the tastes involved tend to support these investigators in their contention that they were observing the consequence of prior exposure to novel tastes per se rather than stimulus generalization; however, the broad generalization across tastes recently observed by Dawley (1979) indicates that stimulus generalization may have influenced some of these studies. Braveman and Jarvis (1978) and Austin (Note 1), using single-bottle tests, found that prior exposures to novel substances reduced neophobia in adult animals. Capretta, Petersik, and Stewart (1975) and Hennessy, Smotherman, and Levine (1977) obtained similar effects in rat pups; however, the former researchers detected no reduction in neophobia in adult rats. The basis for this discrepancy with the other reports is presently unclear, but possibly it originates with the nature of Capretta et al.'s test substance (chocolate extract) or their long test sessions $(24 \mathrm{~h})$ which may have obscured any differential effects of prior gustatory experience. Examining the effects of prior exposure to novel tastes upon CTA acquisition to a dissimilar flavor, Tarpy and McIntosh (1977) found a reduced aversion, whereas Braveman and Jarvis (1978) and Zahorik (1976) failed to observe such a reduction. The basis for this inconsistency is also uncertain, but likely is due to differences in the test trial procedures and differences in the pretraining and test flavors, the latter possibly resulting in more stimulus generalization across flavors in the Tarpy and McIntosh (1977) study. The tendency of rats to generalize across flavors that, to humans, seem highly dissimilar (Dawley, 1979) emphasizes the importance of the particular flavors used for pretraining and training. The present experiments suggest that such stimulus generalization is the most probable basis for the seemingly maladaptive reduction in propensity to form CTAs reported by Tarpy and McIntosh (1977) following exposure to multiple novel flavors.

\section{EXPERIMENT 1}

Our first study was performed to investigate the effect of novel gustatory experiences upon neophobia and CTAs. Previous research on these issues appears, in the light of Dawley's (1979) data, to have possibly been affected by stimulus generalization between flavors. Braveman and Jarvis (1978) made the best effort to date to examine the influence of stimulus generalization in this paradigm. They found that either before or after CTA acquisition, exposure to flavors dissimilar from the CS had no effect upon the CTA; that is, neither latent inhibition of acquisition nor secondary extinction was seen, thereby suggesting that their flavors prohibited significant generalization. However, the very nature of their major conclusion, that different mechanisms underlie CTA and neophobia, raises the possibility that the exclusion of stimulus generalization in respect to CTA does not definitively insure the absence of generalization in respect to neophobia. That observed stimulus generalization might depend upon the response monitored is a concept foreign to the prevailing belief that stimulus generalization arises at some early level of processing, in this case presumably prior to the divergence of information into potentially different pathways (mechanisms) that mediate neophobia and CTA. However, despite the appealing simplicity of this latter view, there are few data to support it. Clearly, stimulus similarity arises in the central nervous system rather than in the receptors. Moreover, the existence of not one but several areas in the brain for processing information arriving in a particular modality admits the possibility of parallel processing and multiple stimulus generalization gradients for a single stimulus dimension. The design of our first experiment was similar to that of Braveman and Jarvis (1978), with the important exception that our flavors were chosen to minimize stimulus generalization between them. In all of the following studies, we attempted to minimize generalization between novel treatment substances and the test substance by restricting ourselves to the use of one substance each that was sweet (sucrose), sour (hydrochloric acid), bitter (quinine), and salty (saline). Using one flavor for testing, this left only three to be used as novel substances. However, the minimizing of stimulus generalization was judged worth this limitation in number of novel tastes. Even with this restricted use of tastes, it is likely that generalization between substances occurred, but there is contemporary evidence indicating that our choice of flavors held such generalization to a minimum (McBurney \& Gent, 1979). Moreover, empirical evidence supporting a lack of stimulus generalization between these four flavors in respect to both neophobia and CTA is presented in Experiments 3 and 4.

\footnotetext{
Method

Subjects and Apparatus. Sixty Sprague-Dawley-derived naive male rats, weighing $200-300 \mathrm{~g}$ on Day 1 of the study, were obtained from a commercial supplier 10-14 days prior to Day 1. Counterbalancing for body weight on the day before treatment began, the subjects were assigned to one of six groups $(n=10)$. All subjects were housed singly in wire-mesh cages and maintained
} 
on ad-lib Purina Lab Chow and water until 7 days prior to Day 1, at which time each animal was put on a $23-h$ /day water-deprivation schedule. Water bottles were placed on the cages $23 \mathrm{~h}$ before any treatment or test and removed $1 \mathrm{~h}$ later; hence, all animals were $22 \mathrm{~h}$ deprived at the time of each flavor exposure, treatment or test. Water bottles were cleaned and refilled daily to prevent the growth of any algae that might alter the taste of the water. Refilled bottles were always permitted to come to room temperature before being given to the animals. All treatments and tests occurred in the home cage. Each novel taste was presented in solution through a lick tube with a small-diameter orifice $(2 \mathrm{~mm})$ that minimized drippage. Absorbent cardboard under each lick tube during treatment and testing confirmed that little drippage occurred. The reservoir behind each lick tube was a specially constructed narrow graduated cylinder that allowed readings accurate to $1 \mathrm{cc}$.

Procedure. Exposure to a flavor consisted of $30 \mathrm{~min}$ of access on each of 4 successive days. Exposure series were separated by 3 days to minimize differences in deprivation at the start of each series. When testing the propensity to form CTAs, only a single training trial and single test trial were used. Moreover, the test trial occurred 3 days after acquisition to permit full recovery from the internal malaise induced by the toxin. Single-bottle tests were used throughout the series in order to avoid the floor effects in CTA that are encouraged by two-bottle tests. The four flavors employed in these experiments were sucrose $(5 \% \mathrm{w} / \mathrm{v})$, $\mathrm{NaCl}(.9 \% \mathrm{w} / \mathrm{v}), \mathrm{HCl}(.01 \mathrm{M}=.036 \% \mathrm{w} / \mathrm{v})$, and anhydrous quinine bisulfate $(.005 \% \mathrm{w} / \mathrm{v}) . \mathrm{LiCl}(.3 \mathrm{M}=1.27 \% \mathrm{w} / \mathrm{v})$ injected intraperitoneally $(.75 \%$ body weight) was the source of internal malaise in the induction of CTA, this relatively low dose having been selected with the intention of avoiding floor effects that might obscure differences between groups in CTA. Animals not receiving $\mathrm{LiCl}$ were injected at the corresponding time with the same volume of $\mathrm{NaCl}(.9 \% \mathrm{w} / \mathrm{v})$ to control for the physical effects of the injection.

One group received ordinary latent inhibition (CS-CS-LiCl) treatment prior to CTA training. On Days 1-4, 7-10, and 13-16, these animals were exposed to $\mathrm{NaCl}$ for $30 \mathrm{~min} /$ day. On Days $5-6,11-12$, and $17-18$, this group received only the $1 \mathrm{~h}$ of water that occurred each and every day. On Day 19, the conditioning day, these animals were again exposed to $\mathrm{NaCl}$ for $30 \mathrm{~min}$, and $10 \pm 2$ min later were injected with $\mathrm{LiCl}$. To test for the acquisition of $\mathrm{CTA}, \mathrm{NaCl}$ was once again presented for $30 \mathrm{~min}$ on Day 22.

A second group was exposed to diverse novel tastes (DNT$\mathrm{CS}-\mathrm{LiCl}$ ) prior to CTA training. These animals were given sucrose on Days 1-4, quinine on Days 7-10, $\mathrm{HCl}$ on Days 13-16, and $\mathrm{NaCl}$ on Days 19 and 22. Like Group CS-CS-LiCl, this group was injected with $\mathrm{LiCl} 10 \pm 2$ min after the end of the $\mathrm{NaCl}$ exposure of Day 19. A third group (DNT-CS-Sal) was treated identically to Group DNT-CS-LiCl except that it received a saline injection in place of the $\mathrm{LiCl}$.

The remaining three control groups received no treatments until Day 19. The first of these groups (CS-LiCl) was given $\mathrm{NaCl}$ and injected with $\mathrm{LiCl}$ on Day 19, and was tested with $\mathrm{NaCl}$ on Day
22. The second group ( $\mathrm{LiCl}-\mathrm{CS}$ ) received the same treatment as Group $\mathrm{CS}-\mathrm{LiCl}$ except that the $\mathrm{LiCl}$ injections were given $4 \mathrm{~h}$ earlier, thereby constituting a backwards conditioning paradigm with a long interstimulus interval. The last group (CS-Sal) was also treated the same as Group $\mathrm{CS}-\mathrm{LiCl}$, except that on Day 19 it was injected with physiological saline rather than $\mathrm{LiCl}$. Table 1 summarizes the treatments of the different groups.

\section{Results and Discussion}

Although prior exposure to the $\mathrm{CS}(\mathrm{NaCl})$ yielded latent inhibition (CS-CS- $\mathrm{LiCl}$ vs. $\mathrm{CS}-\mathrm{LiCl}$ on Day 22 ), prior exposure to diverse novel flavors did not significantly interfere with acquisition of the CTA (DNT-CS-LiCl vs. CS-LiCl on Day 22). However, this prior exposure to novel flavors did reduce neophobia to $\mathrm{NaCl}$ (DNT-CS-LiCl and DNT-CS-Sal vs. $\mathrm{CS}-\mathrm{LiCl}$ and CS-Sal on Day 19). The mean consumption scores are given in Figure 1.

Analysis of variance of consumption data for the six groups on the neophobia test (Day 19) and the CTA test (Day 22) both proved significant $[F(5,54)$ $=2.48, p<.05$, and $F(5,54)=8.78, p<.001$, respectively]. (Replication of the critical results of this study in Experiments 2-4 speaks for the reliability of the present findings.) Between-group comparisons of neophobia test day consumption (Day 19) found significantly greater consumption by Groups CS-CS-LiCl and DNT-CS-Sal than by Groups CS-LiCl and CS-Sal [all ts $(18)>2.37$, ps $<.05$ ], suggesting that prior exposure to either the $\mathrm{CS}(\mathrm{NaCl})$ or diverse novel stimuli had reduced neophobia. The greater consumption by Group CS-CS- $\mathrm{LiCl}$ than by Groups DNT-CS-LiCl or DNT-CS-Sal did not prove significant [both $\mathrm{ts}(18)<.62$, ps $>.5$ ]; however, the tendency suggests less neophobia in animals that had previously been exposed to $\mathrm{NaCl}$ than in those animals that had had prior experience with diverse novel stimuli. The only other significant difference on Day 19 was that Group LiCl-CS drank less $\mathrm{NaCl}$ than any other group [all ts $(18)>2.41$, ps $<.05$ ], an effect that perhaps resulted from these animals' being slightly ill or unusually wary as a consequence of having been poisoned $4 \mathrm{~h}$ earlier.

Between-groups comparisons of CTA test day consumption (Day 22) found that Groups DNT-CS-

Table 1

Treatment Conditions Used in Experiment 1

\begin{tabular}{llcccc}
$\begin{array}{c}\text { Group } \\
\text { Designation }\end{array}$ & Days 1-4 & Days 7-10 & Days 13-16 & Day 19 & Day 22 \\
\cline { 2 - 5 } CS-CS-LiCl & $\mathrm{NaCl}$ & $\mathrm{NaCl}$ & $\mathrm{NaCl}$ & $\mathrm{NaCl-LiCl}$ & $\mathrm{NaCl}$ \\
DNT-CS-LiCl & Sucrose & Quinine & $\mathrm{HCl}$ & $\mathrm{NaCl}-\mathrm{LiCl}$ & $\mathrm{NaCl}$ \\
DNT-CS-Sal & Sucrose & Quinine & $\mathrm{HCl}$ & $\mathrm{NaCl}-\mathrm{Saline}$ & $\mathrm{NaCl}$ \\
CS-LiCl & & & & $\mathrm{NaCl}-\mathrm{LiCl}$ & $\mathrm{NaCl}$ \\
LiCl-CS & & & $\mathrm{LiCl}-\mathrm{NaCl}$ & $\mathrm{NaCl}$ \\
CS-Sal & & & $\mathrm{NaCl}-\mathrm{Saline}$ & $\mathrm{NaCl}$ \\
\hline
\end{tabular}

Note-Each exposure to a flavored solution lasted $30 \mathrm{~min}$. All injections on Day 19 followed removal of the $\mathrm{NaCl}$ by $10 \pm 2 \mathrm{~min}$, except for Group LiCl-CS, which received its injection $4 \mathrm{~h}$ prior to the $\mathrm{NaCl}$. 


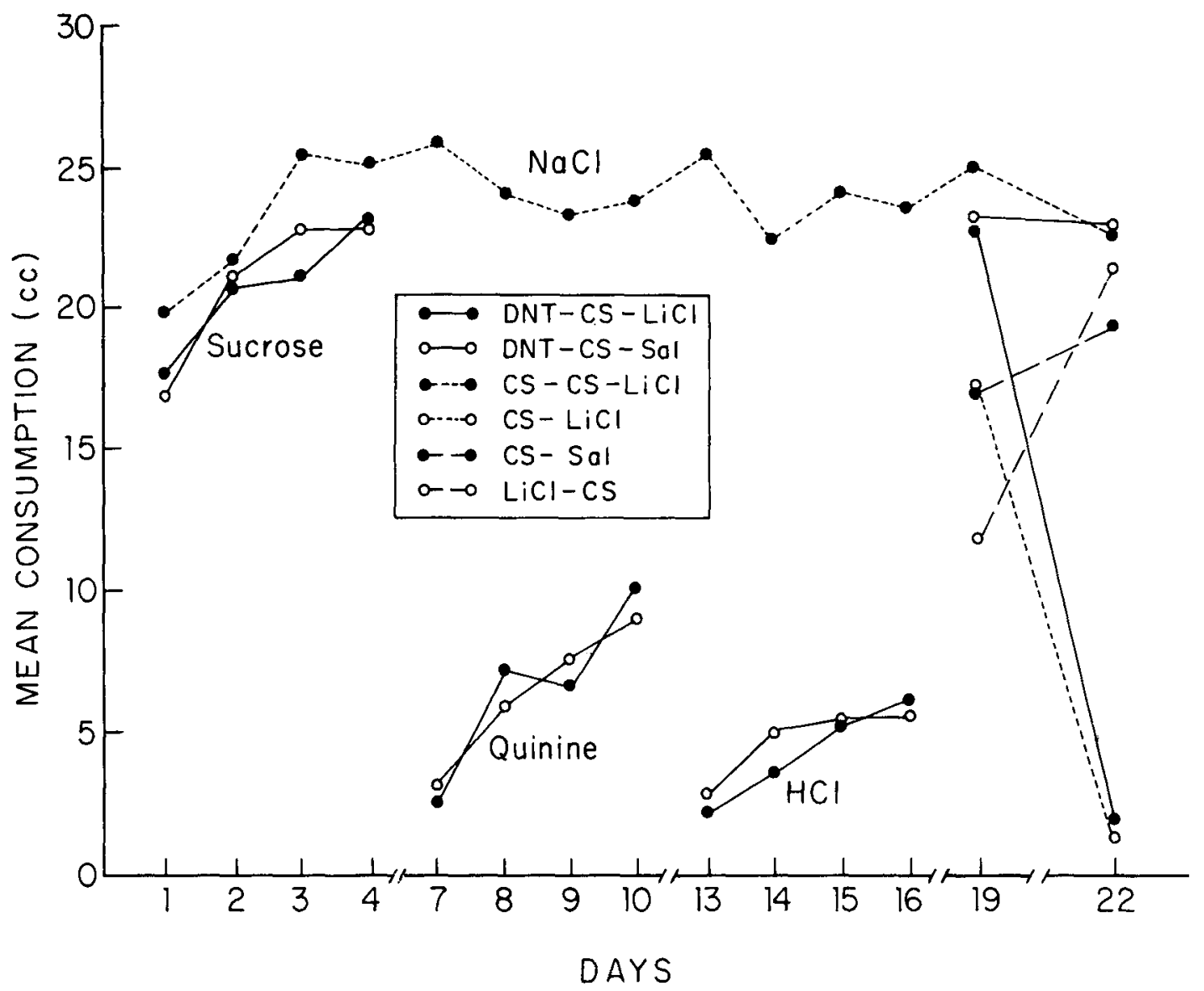

Figure 1. Daily group mean consumption during 30-min trials in Experiment 1 ( $\mathrm{n}=10$ ). On Days 19 (training) and 22 (testing), consumption was of the $\mathrm{CS}, \mathrm{NaCl}$, for all groups.

$\mathrm{LiCl}$ and $\mathrm{CS}-\mathrm{LiCl}$, which did not differ $[\mathrm{t}(18)=.36$, $\mathrm{p}>.5$, consumed less $\mathrm{NaCl}$ than Groups CS-CSLiCl, DNT-CS-Sal, LiCl-CS, and CS-Sal [all ts(18) $>2.61$, ps $<.02$ ]. There were no differences among the last four groups [all ts $(18)<1.70$, ps $>.10$ ]. The high consumption of the two groups injected with saline indicates that suppression in Groups DNT$\mathrm{CS}-\mathrm{LiCl}$ and $\mathrm{CS}-\mathrm{LiCl}$ was a consequence of the internal malaise induced by $\mathrm{LiCl}$. And the relatively high consumption of Group $\mathrm{LiCl}-\mathrm{CS}$ demonstrates that the intake suppression in Groups DNT-CS-LiCl and $\mathrm{CS}-\mathrm{LiCl}$ is associative, rather than due to, sensitization or pseudoconditioning resulting from exposure to $\mathrm{LiCl}$. The large intake of Group CS$\mathrm{CS}-\mathrm{LiCl}$ may reasonably be attributed to latent inhibition of CTA acquisition.

Examining the data for decreased neophobia as measured by increases in consumption over repeated exposures to an initially novel substance, withinsubjects tests between consumption on the first and fourth days of exposure yielded nearly significant reductions in neophobia for $\mathrm{NaCl}$ in Group CS-CS$\mathrm{LiCl}[\mathrm{t}(9)=1.96, .05<\mathrm{p}<.10]$ and clear reductions of neophobia in the cases of sucrose, quinine, and HCL (Groups DNT-CS-LiCl and DNT-CS-Sal) [all ts $(19)>2.19$, ps $<.05$ ]. Whether the withingroups neophobias measured later in the treatment sequence, such as that for $\mathrm{HCL}$, were reduced by prior treatment with dissimilar novel tastes cannot be answered in the present study, but is addressed in Experiment 2. Similarly, the possible effects of Day 19 consumption differences on CTA acquisition are discussed following Experiment 2.

The present study included no measure of the stimulus specificity of the CTA acquired after a single $\mathrm{NaCl}-\mathrm{LiCl}$ pairing. To investigate this issue, a small follow-up study was conducted with two groups $(\mathrm{n}=9)$, one being treated identically to Group CS-LiCl on Day 19 and the other being treated the same as Group CS-Sal. However, 3 days later, both groups were tested with sucrose rather than the $\mathrm{CS}(\mathrm{NaCl})$. On the training day, the former group yielded a mean of $16.0 \mathrm{cc}$ and the latter group had a mean of $16.4 \mathrm{cc}$. On the test day, the former (poisoned) group consumed a mean of $15.2 \mathrm{cc}$ of sucrose and the latter (control) group consumed a mean of $18.0 \mathrm{cc}$ of sucrose. This difference was not significant $[\mathrm{t}(16)=1.01, \mathrm{p}>.2]$. Although there was a nonsignificant trend toward increased neophobia following a $\mathrm{NaCl}-\mathrm{LiCl}$ pairing, the size of 
this effect was small compared with the strong rejection of the CS on Day 22 by Groups DNT-CS-LiCl and $\mathrm{CS}-\mathrm{LiCl}$. These results suggest that the rejection of $\mathrm{NaCl}$ Day 22 seen in Experiment 1 reflected CTAs relatively specific to the $\mathrm{CS}$ that preceded $\mathrm{LiCl}$ rather than an enhanced neophobia, although the nonsignificant trend in the follow-up study is consistent with other studies that have obtained illness-induced enhanced neophobia (e.g., Best \& Batson, 1977).

In summary, the present experiment obtained the same basic effects as Braveman and Jarvis (1978) and, by virtue of the flavors used, reduced the likelihood that stimulus generalization contributed to the observed reduction in neophobia. Thus, the seeming discrepancy between the hypothesized protective role of neophobia (Barnett \& Cowan, 1976) and the ease with which neophobia can be reduced without exposure to the flavor in question appears to be quite real, and suggests that neophobia plays at most a slight protective role. Moreover, the present change in neophobia without any modification in capacity to form a CTA suggests that the two phenomena have different bases. As CTAs appear to have a defensive value in respect to ingestion, the lack of correspondence between changes in CTA potental and neophobia is consistent with neophobia's not being a primary defense against poisoning. This last conclusion must be tempered by the possibility that the Day 22 comparison of CTA between Group CS$\mathrm{LiCl}$ and Group DNT-CS-LiCl was not sensitive. One potential problem is that a floor effect suppressed real differences. This possibility cannot be categorically rejected on the basis of the present data; however, all but one animal in Groups CS-LiCl and DNT$\mathrm{CS}-\mathrm{LiCl}$ drank appreciable amounts $(>.2 \mathrm{cc})$ on Day 22, and both groups differed significantly from zero intake $[\mathrm{t}(9)=2.96, \mathrm{p}<.02$, and $\mathrm{t}(4)=3.30$, $\mathrm{p}<.01$, respectively]. (See Experiment 5 for further evidence against a floor effect obscuring a difference in CTAs.) A second possibility was suggested by Braveman and Jarvis (1978): one-bottle tests are differentially sensitive to neophobia and CTA. Such differential sensitivity would also suggest different mechanisms underlying neophobia and CTAs. However, no divergence between observed neophobia and CTA potential can definitively establish the existence of different underlying mechanisms rather than merely different response rules.

\section{EXPERIMENT 2}

The previous study suggested that neophobia to $\mathrm{NaCl}$, but not the propensity to form an association between $\mathrm{NaCl}$ and $\mathrm{LiCl}$, was reduced through prior exposure to dissimilar novel tastes. These results are incongruent with the proposal of Best (1975) that reductions in neophobia to a particular substance ordinarily reflect learning that the substance does not have significant consequences (latent inhibition), a state that is diametrically opposed to, and should therefore retard acquisition of, a CTA for the substance. The failure of prior exposure to diverse novel tastes to attenuate CTA suggests that learned irrelevance is not capable of explaining all reductions in neophobia. The apparent need for an additional mechanism was disturbing and caused us in Experiment 2 to examine the generality of the results of the first study by using test substances other than $\mathrm{NaCl}$.

\section{Method}

Subjects and Apparatus. Ninety-six rats identical in origin, maintenance, and size to those used in Experiment 1 were assigned to one of eight groups on a random basis after counterbalancing for body weight $(n=12)$. The experimental setting and solution concentrations were unchanged from the previous experiment.

Procedure. Four groups were treated identically to Group DNT$\mathrm{CS}$ - $\mathrm{LiCl}$ in Experiment 1 except that, for one group, $\mathrm{HCl}$ served as the test flavor on Days 19 and 22 (DNT-HCl); for a second group, sucrose was the test flavor (DNT-Suc); for a third group, quinine served as the test flavor (DNT-Quin); and for the fourth group, $\mathrm{NaCl}$ was the test flavor (DNT-NaCl). Each group's pretraining exposure was to three of the four flavors ( $\mathrm{NaCl}$, sucrose, $\mathrm{HCl}$, and quinine), with the test substance for that group exciuded. Moreover, to avoid any effects due to the order of the pretraining exposures, each of these four groups was further divided into six subgroups $(n=2)$. The six subgroups represented all possible permutations of order of the pretreatment flavors. Since the subgroups were so small in size, no analysis was performed on their separate scores; however, their presence assured that the present results were not unique to one or another order of presentation of the three pretraining flavors.

The other four groups included in this study received exactly the same treatment as Group $\mathrm{CS}-\mathrm{LiCl}$ in Experiment 1 except that one group received $\mathrm{HCl}$ as the test flavor on Days 19 and 22 ( $\mathrm{HCl}$ ), a second received sucrose (Suc), a third received quinine (Quin), and the fourth received $\mathrm{NaCl}(\mathrm{NaCl})$. In light of the data from Experiment 1, nonpoisoned control groups and backwards or explicitly unpaired control groups were not included in the present study.

\section{Results and Discussion}

Relative to animals with no previous flavor experience, prior exposure to novel flavors different from that used in testing reduced Day 19 neophobia to the test substance when the test flavor was $\mathrm{NaCl}$ or $\mathrm{HCl}$, but did not do so significantly when the test flavor was sucrose or quinine. Day 22 consumption scores indicated that in no instance was acquisition of the CTA significantly attenuated. Group means for Days 19 and 22 are presented in Figure 2. Days $1-4,7-10$, and $13-16$ in Figure 2 reflect the pooling across treatment groups of subgroups that received the same flavor on that set of 4 days.

A 2 by 4 (Prior Diverse Novel Tastes by Test Flavor) analysis of variance on Day 19 consumption scores yielded a significant effect for DNT [F $(1,88)$ $=4.05, p<.05]$, flavor $[F(3,88)=14.94, p<.001]$, and their interaction $[F(3,88)=2.73, p<.05]$. A similar analysis of Day 22 scores yielded no significant differences [all Fs $<1.92$, ps $>.10$ ].

Comparisons between individual groups on Day 19 


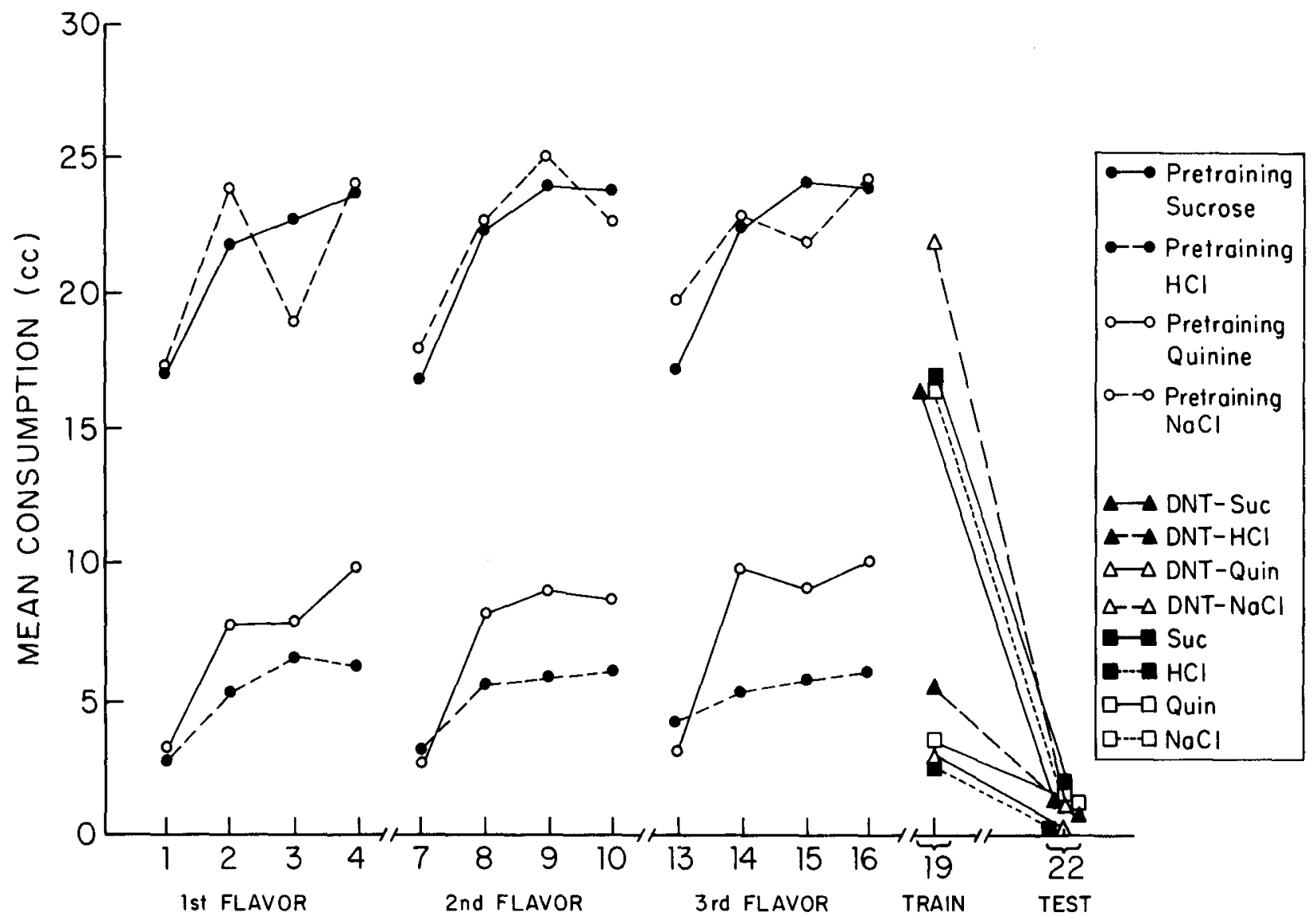

DAYS

Figure 2. Daily group mean consumption during 30-min trials on Days 19 and 22 in Experiment 2 (n = 12). The data for Days 1-4, 7.10, and 13-16 represent means of 12 animals consisting of four subjects from each DNT group scheduled for exposure to the indicated substance prior to training. All subjects were poisoned with $\mathrm{LiCl}$ after ingesting their test substance on Day 19 (training).

found Group DNT-NaCl to consume more than Group $\mathrm{HCL}[\mathrm{t}(22)=2.11, \mathrm{p}<.05]$ and Group DNT-NaCl to drink more than Group $\mathrm{NaCl}[\mathrm{t}(22)=2.19, \mathrm{p}<$ $.05]$, but no differences between the two sucrose groups $[\mathrm{t}(22)=.43, \mathrm{p}>.50]$ or the two quinine groups $[\mathrm{t}(22)=.51, \mathrm{p}>.50]$. These results suggest that prior exposure to diverse novel tastes reduced neophobia to $\mathrm{NaCl}$ (consistent with Experiment 1 and Braveman \& Jarvis, 1978) and $\mathrm{HCl}$, but not to sucrose or quinine. Consistent with a loss of neophobia toward $\mathrm{NaCl}$ and $\mathrm{HCl}$ are the tendencies toward increasing first day consumption of $\mathrm{NaCl}$ and $\mathrm{HCl}$ over their presentations as the first, second, third, and fourth flavors, tendencies not seen in sucrose or quinine (see Figure 2); however, none of these tendencies proved significant until the fourth flavor was introduced on Day 19.

As foreshadowed by the analysis of variance of Day 22 consumption scores, no differences between Day 22 scores of groups tested on the same substance proved significant [all ts $(22)<.73$, ps $>.20$ ]. Moreover, all groups yielded a significant decrement in consumption between Day 19 and Day 22 (all ts(11)
$>2.29$, ps $<.05]$. These results suggest that a CTA was acquired to all four flavors regardless of prior experience with dissimilar novel flavors and regardless of whether or not exposure to diverse novel tastes produced reductions in neophobia. The present failure to interfere with formations of CTAs is particularly notable in light of the successful demonstration of latent inhibition in Experiment 1 (Group CS-CS-LiCl), which speaks for the sensitivity of the current preparation to impairments of CTA acquisition.

Three notes of caution are necessary in interpreting the present data. The first concerns the observation of prior experience with diverse novel tastes reducing neophobia to some substances $(\mathrm{HCl}$ and $\mathrm{NaCl})$ but not to others (sucrose and quinine). This finding may depend on the concentration of each substance used. While the present effects are all relative to nonDNT control groups that received the same test flavor and concentration, it is still possible that all DNT groups underwent a decrement in neophobia, but that the concentration of sucrose and quinine was too great for this attenuation to be evidenced, that is, 
changes in neophobia for sucrose and quinine may have been obscured by a ceiling effect for manifest neophobia. However, the likelihood appears small that this was the sole basis of the differences in neophobia reduction between test substances as a result of prior exposure to diverse novel tastes. Notably, $\mathrm{HCl}$ and quinine were of such concentrations that they were comparable in palatability (Groups Quin and $\mathrm{HCl}$ ), as were the concentrations of sucrose and $\mathrm{NaCl}$ (Groups Suc and $\mathrm{NaCl}$ ); yet, one member of each of these pairs yielded a reduction in neophobia while the other did not. Nevertheless, these comparisons between effects on neophobia to differing tastes should be regarded with caution until comprehensive parametric studies across test flavor concentration levels have been performed.

Caution is also appropriate in interpreting the lack of effects of prior DNT upon the acquisition of CTAs to $\mathrm{HCl}$ and $\mathrm{NaCl}$, as differential amounts of these substances as a function of prior exposure to diverse novel tastes were consumed on the conditioning day, Day 19. On the one hand, the heightened consumption by Groups DNT-HCl and DNT-NaCl compared with Groups $\mathrm{HCl}$ and $\mathrm{NaCl}$ provided higher baselines from which CTAs must depart. This inequity is a potential source of bias toward Group DNT-NaCl's and Group DNT-HCl's manifesting greater consumption than controls on Day 22 even if CTA acquisition were equal. However, as no such differences appeared on Day 22, this possible confound either failed to arise or was inconsequential in magnitude. On the other hand, the greater consumption by Groups DNT- $\mathrm{HCl}$ and DNT $-\mathrm{NaCl}$ than by $\mathrm{Groups} \mathrm{HCl}$ and $\mathrm{NaCl}$, respectively, might lead one to expect superior CTA acquisition, as greater CS consumption on the conditioning trial can result in better CTA acquisition (Bond \& DiGiusto, 1975; Braveman \& Crane, 1977). This tendency is in opposition to prior DNTs' reducing any subsequent CTA, such as reported by Tarpy and McIntosh (1977), and hence could have masked any direct effect of prior DNT upon CTA. However, there are data that argue against the notion that a CS magnitude effect on conditioning obscures any potential attenuation in CTA acquisition. Several researchers have reported a CTA enhancement ceiling due to CS magnitude effects' being reached with consumption of surprisingly low volumes, particularly with short CS-US intervals such as the $10 \mathrm{~min}$ of the current research (Barker, 1976; Braveman \& Crane, 1977; Deutsch, 1978). In the present study, Day 19 consumption of $\mathrm{NaCl}$ by both Groups DNT-NaCl and $\mathrm{NaCl}$ was greatly in excess of what one would expect for a CS magnitude effect; hence, these groups are not likely to have been subject to this potential confound. The problem remains, however, for CTA acquisition to $\mathrm{HCl}$, unless one accepts Kalat's (1976) conclusion that CS volume consumed is a minor, if not irrelevant, variable in CTA acquisition.
A final qualification in interpreting the effects of prior experience with diverse novel tastes upon CTA acquisition arises from the low absolute scores of all groups on Day 22. There is the possibility that potential differences in associative strength were hidden by a floor effect. We are not inclined to give much weight to this possibility, as all but two of the 96 subjects drank measurable amounts of their test substance $(>.2 \mathrm{cc})$. Further arguing against a floor effect in any group on Day 22 is the finding that all groups differed from zero intake (all ts $(11)>3.08$, ps $<.02)$. However, the possibility cannot be categorically rejected until the potential floor effect has been eliminated through a reduction in the amount of $\mathrm{LiCl}$ injected. (See Experiment 5 for evidence against an artificial equality in CTA for the salty and sour solutions arising from a floor effect.)

Despite the foregoing qualifications, we presently favor the conclusion that prior DNT attenuated neophobia to $\mathrm{NaCl}$ and $\mathrm{HCl}$ but not sucrose and quinine, and that it did not affect acquisition of CTAs. One possible explanation of these differential changes in neophobia as a function of test flavors is that, despite the difference in sensitivity of receptors to $\mathrm{HCl}$ and $\mathrm{NaCl}$ (McBurney \& Gent, 1979), these two substances generalized sufficiently to each other such that exposure to one of these flavors reduced neophobia to the other, whereas neither flavor generalized to sucrose or quinine, which did not generalize to each other. This view, however, is viable only if separate mechanisms with different stimulus generalization gradients are assumed to underlie neophobia and CTA. Experiment 3 examines, among other matters, the possibility of generalization between $\mathrm{HCl}$ and $\mathrm{NaCl}$.

\section{EXPERIMENT 3}

Although the effects observed in Experiment 2 were attributed to prior exposure to diverse novel tastes, no control group was included to examine the likelihood that a single or repeated prior exposure to any one of the pretraining tastes might have been adequate to produce the observed decrements in neophobia to $\mathrm{HCl}$ and $\mathrm{NaCl}$. Experiment 3 investigated this possibility, focusing specifically upon the sequence of treatments (first sucrose, then quinine, and finally $\mathrm{HCl}$ ) that attenuated neophobia to $\mathrm{NaCl}$ in Group DNT-CS-LiCl of Experiment 1.

A second rationale for Experiment 3 was to further examine the possibility that stimulus generalization between $\mathrm{HCl}$ and $\mathrm{NaCl}$ underlies the reduced neophobia to $\mathrm{NaCl}$ and $\mathrm{HCl}$ seen in Experiment 2. Two factors argue against generalization between our salty and sour solutions: (1) our choice of flavors for pretraining exposure and training based upon the distinctiveness of these flavors as discussed by McBurney and Gent (1979), and (2) the lack of attenuated CTAs due to generalized latent inhibition. However, the 
possibility cannot be categorically rejected that, rather than exposure to diverse tastes per se, latent inhibition during pretraining exposures and flavor generalization at the time of neophobia testing collectively produced the observed reductions in neophobia. The lack of latent inhibition of CTA could stem from the generalization gradient measured by CTA being narrower than the generalization gradient for neophobia. If generalization between one or more of the pretraining flavors and the flavor used in training is responsible for the observed reduction in neophobia, then tripling the exposure to one pretraining substance while eliminating pretraining exposure to the other two pretraining substances ought to unambiguously determine if the remaining pretraining flavor generalizes to the training flavor as indexed by reduced neophobia. Similarly, extended exposure to the pretraining flavor ought to yield some degree of latent inhibition of CTA formation to the training flavor if there were any stimulus generalization between flavors. Experiment 3 included groups sensitive to any such effects.

\section{Method}

Subjects and Apparatus. Eighty naive male rats similar in origin, maintenance, and size to those of the previous studies were as- signed randomly to one of eight groups $(n=10)$ after counterbalancing for body weight. The apparatus and fluid concentrations were identical to those used in Experiments 1 and 2.

Procedure. Except as noted, all groups in this study received the same treatment as Group DNT-CS-LiCl of Experiment 1 (see Table 1). Group Suc-Quin-HCl was an exact replication of that prior group. Three groups were included to determine if the reduced neophobia anticipated in Group Suc-Quin-HC1 was merely the algebraic sum of separate exposures to sucrose, quinine, and $\mathrm{HCl}$ rather than due to the diversity of past flavor experience: Group Suc-NT-NT received 30 min of sucrose on Days 1-4 and no treatment on Days 5-18; Group NT-Quin-NT was given quinine on Days 7.10 and no novel flavor on Days 1-6 and 11-18; and Group NT-NT-HCl received no treatment on Days $1-12$ and $\mathrm{HCl}$ on Days 13-16. Another three groups were included to probe the possibility that frequency of prior exposures, but not diversity, was of consequence, and also to enhance any possible effects of generalization between flavors: Group 3Suc received $30 \mathrm{~min}$ of sucrose on Days 1-5, 7-10, and 13-16; Group 3Quin was given quinine on the same 12 days; and Group $3 \mathrm{HCl}$ received $\mathrm{HCl}$ on all of these days. Group 3NT was a no-treatment control group that received nothing but the food and water of the maintenance regimen on Days 1-18. All eight groups were of fered $\mathrm{NaCl}$ and then injected with $\mathrm{LiCl}$ on Day 19 and tested for $\mathrm{NaCl}$ consumption on Day 22. No nonpoisoned control animals were deemed necessary since such groups had been included in Experiment 1 and had behaved appropriately.

\section{Results and Discussion}

As shown in Figure 3, pretraining treatment with all three flavors dissimilar to the CS attenuated neo-

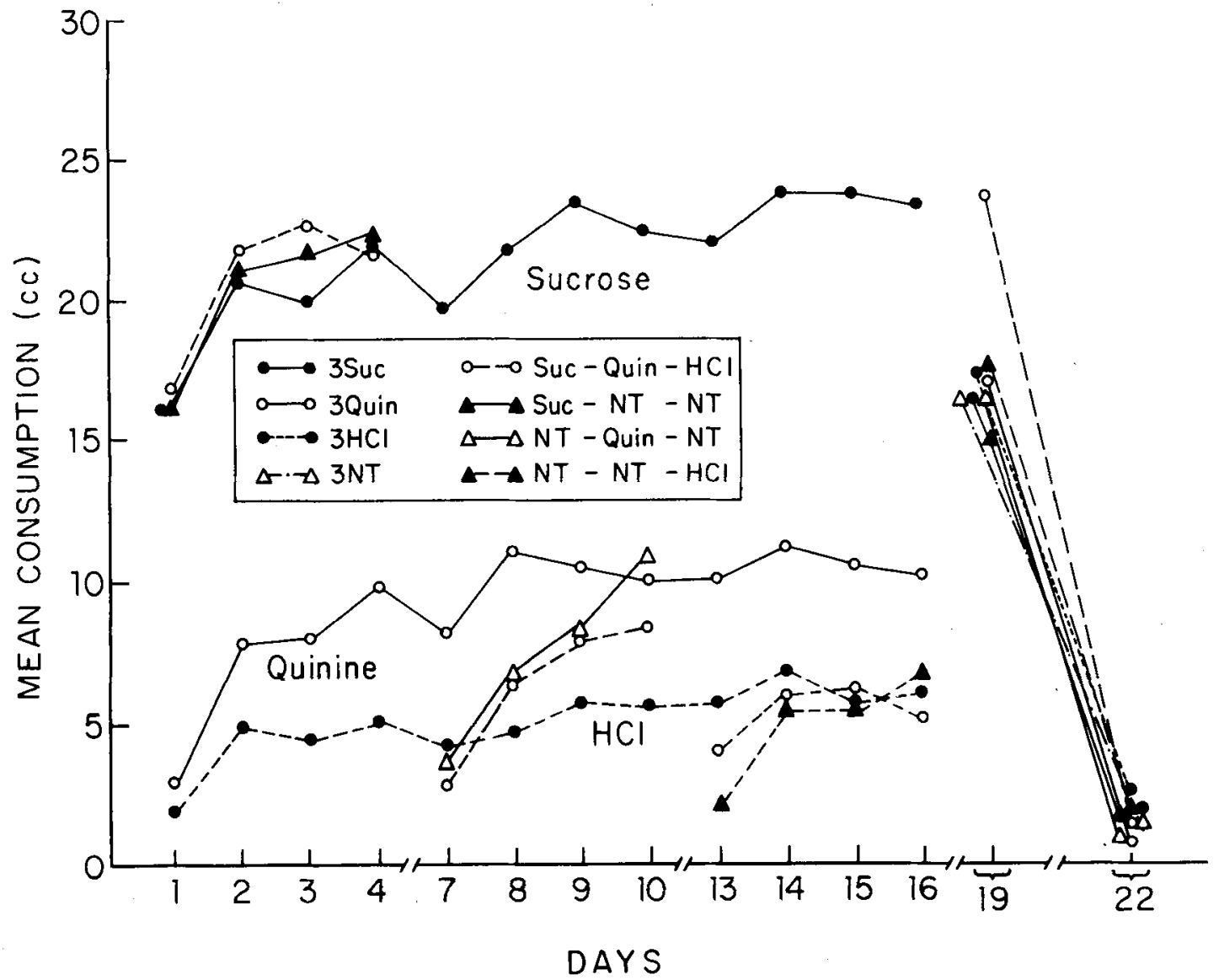

Figure 3. Daily group mean consumption during 30-min trials in Experiment $3(n=10)$. On Days 19 (training) and 22 (testing), consumption is of the $\mathrm{CS}, \mathrm{NaCl}$, for all groups. All animals were injected with LiCl after their first exposure to $\mathrm{NaCl}$, which occurred on Day 19. 
phobia to $\mathrm{NaCl}$, whereas prior exposure to only one of the three dissimilar flavors either for 4 or 12 days failed to reduce neophobia. None of the treatments interfered with the acquisition of the $\mathrm{NaCl}-\mathrm{LiCl}$ association.

One-way analysis of variance of Day 19 consumption scores approached significance $[F(7,72)=1.97$, $.05<\mathrm{p}<.10]$. Individual comparisons between Group 3NT, the basic control group, and each other group found a difference relative to Group Suc-Quin$\mathrm{HCl}[\mathrm{t}(18)=2.60, \mathrm{p}<.02]$, but not to any other group [all ts $(18)<.71$, ps $>.2$ ]. Further analyses established that Group Suc-Quin- $\mathrm{HCl}$ differed from all other groups [all ts $(18)>2.16$, ps $<.05$ ], whereas all other groups failed to differ from each other [all ts(18) $\langle 1.24$, ps $>$.2]. The failure of Groups 3Suc, 3Quin, and particularly $3 \mathrm{HCl}$ to distinguish themselves from Group 3NT contrasts sharply with the difference observed between Groups Suc-Quin-HCl and 3NT, that is, reduced neophobia, and suggests that the latter difference is not due to any generalization of reduced neophobia upon a taste similarity dimension. Rather, these results lend support to the interpretation that the reduction in neophobia seen in Experiments 1-3 following exposure to novel tastes dissimilar to the test substance is dependent upon the diversity of the pretraining flavors instead of the action of any one flavor in particular. Moreover, the observed decrease in neophobia by Group Suc-Quin- $\mathrm{HCl}$ appears to be more than the algebraic sum of decreases produced by each of the three pretraining flavors separately, and this appears to be true even when number of exposures to a flavor is tripled over that of Group Suc-Quin-HCl. Although this last statement is dependent upon the transformation of neophobia into Day 19 consumption scores, the fact that Group 3NT scored near the averages of the other groups, except Group Suc-Quin$\mathrm{HCl}$, indicates that a summation hypothesis would require a complex nonlinear transformation.

Similar analyses of Day 22 scores yielded neither overall significance $[F(7,72)=1.10, p>.25]$ nor significant differences between any two groups [all ts $(18)<1.59$, ps $>.10$ ]. As is apparent in Figure 3, all groups yielded a significant decrease in consumption between Day 19 and Day 22 [all ts(19) $>3.81$, ps $<.005$ ], indicative of strong CTAs. Moreover, the lack of differences between CTAs seen on Day 22 is not likely to be due to a floor effect, because all groups differed from zero intake [ts $(9)>2.78$, ps $<.05$ ].

Consistent with the conclusion of Experiment 2 that prior exposure to dissimilar flavors decreases neophobia to $\mathrm{HCl}$, on Day 13 intake of $\mathrm{HCl}$ was significantly greater in Group Suc-Quin- $\mathrm{HCl}$ than in Group NT-NT-HCl $[\mathrm{t}(18)=2.28, \mathrm{p}<.05]$.

\section{EXPERIMENT 4}

Our interpretation of the results of Experiments 1-3 presumes that the reduced neophobia to our salty and sour solutions was due to prior exposures to diverse novel flavors rather than stimulus generalization between these two flavors. The basis to date for our tentative rejection of generalization between $\mathrm{NaCl}$ and $\mathrm{HCl}$ is threefold: (1) the distinctiveness of these flavors in both humans and infrahuman mammals (McBurney \& Gent, 1979), (2) the lack of cross-taste latent inhibition of CTA formation seen in Experiments 1,2 , and especially 3 (sensitivity to withinflavor latent inhibition was demonstrated in Experiment 1), and (3) the lack of any attenuation of neophobia to $\mathrm{NaCl}$ despite 12 prior exposures to $\mathrm{NaCl}$ in Experiment 3 (sensitivity to within-flavor attenuation of neophobia to $\mathrm{NaCl}$ was also demonstrated in Experiment 1). Despite this evidence against stimulus generalization between our salty and sour solutions, a need was felt for yet further evidence concerning the possibility of generalization between these flavors.

One simple way to detect the existence of any generalization between our $\mathrm{NaCl}$ and $\mathrm{HCl}$ solutions would be to pair one flavor with poison and then test for attenuated intake of the other flavor. However, poisoning is known to influence subsequent ingestion of novel substances; moreover, controlling for this nonassociative effect is difficult, because the effect apparently varies as a function of the poison's being paired with any (different) novel taste (e.g., Best \& Batson, 1977). Therefore, following the lead of Best and Batson (1977) and Domjan (1975) in measuring generalization between flavors, we elected to use an extinction paradigm to further test for generalization. Specifically, all animals were exposed to one of the two flavors, followed soon afterward by an injection of $\mathrm{LiCl}$. Subsequently, extinction exposures were given to half the animals. Following this, we examined acceptance of the other flavor, that is, the flavor not previously experienced. Any generalization between the two flavors should appear as a greater acceptance of the novel test flavor by the extinguished animals than by the nonextinguished animals. Essentially, this test for generalization takes advantage of the heightened neophobia for the novel flavor produced by $\mathrm{LiCl}$, and then measures, across flavors, generalization of extinction to aversiveness of the CS. Finally, all animals were tested on the original flavor to assure that a CTA had been established and that extinction had in fact occurred.

\section{Method}

Subjects and Apparatus. Forty-eight naive rats identical in origin, maintenance, and size to those used in Experiment 1 were assigned to one of four groups on a random basis after counterbalancing for body weight $(n=12)$. The experimental setting and solution concentrations were the same as in the previous experiments.

Procedure. Groups were designated on the basis of the flavor to which they were exposed in conjunction with poison and whether or not they received extinction treatment. Groups $\mathrm{NaCl}$ and $\mathrm{NaCl}-\mathrm{E}$ were given access to the $\mathrm{NaCl}$ solution for $30 \mathrm{~min}$. Simultaneously, Groups $\mathrm{HCl}$ and $\mathrm{HCl}-\mathrm{E}$ were given access to the $\mathrm{HCl}$ 
solution. Then $10 \pm 2$ min later, all animals were injected with $\mathrm{LiCl}$. Following a 3-day interval, during which the animals were allowed to recover from the systemic effects of the poison, Groups $\mathrm{NaCl}-\mathrm{E}$ and $\mathrm{HCl}-\mathrm{E}$ were given $24 \mathrm{~h}$ of access to $\mathrm{NaCl}$ and $\mathrm{HCl}$, respectively. Groups $\mathrm{NaCl}$ and $\mathrm{HCl}$ received ad-lib water during this time. Then, after a 3-day interval on the initial restricted water regimen, Groups $\mathrm{NaCl}$ and $\mathrm{NaCl}-\mathrm{E}$ were given 30 min access to $\mathrm{HCl}$ while Groups $\mathrm{HCl}$ and $\mathrm{HCL}-\mathrm{E}$ were given equal access to $\mathrm{NaCl}$. After another 3-day interval on the restricted water regimen, all animals were given a final 30 -min test, this time on the flavored solution they had received on the conditioning day.

\section{Results and Discussion}

Looking first at the data from the second test, Group $\mathrm{NaCl}$ drank an average of $1.6 \mathrm{cc}$ of the $\mathrm{NaCl}$ solution, which was less than the average of $14.6 \mathrm{cc}$ consumed by Group NaCl-E [t(22) $=2.78, \mathrm{p}<.02]$. Similarly, Group $\mathrm{HCl}$ drank an average of .6 cc of the $\mathrm{HCl}$ solution, which was less than the average of $4.1 \mathrm{cc}$ consumed by Group HCl-E $[\mathrm{t}(22)=2.13$, $\mathrm{p}<.05$ ]. Collectively, these two differences indicate that the extinction treatments were highly effective in reducing the aversion to the flavor paired with $\mathrm{LiCl}$.

Turning to the first test data, Group $\mathrm{NaCl}$ drank an average of $3.8 \mathrm{cc}$ of the $\mathrm{HCl}$ solution, which did not differ from the average of $4.3 \mathrm{cc}$ consumed by Group NaCl-E $[\mathrm{t}(22)=.87, \mathrm{p}>.2]$. Similarly, Group $\mathrm{HCl}$ drank an average of $15.9 \mathrm{cc}$ of the $\mathrm{NaCl}$ solution, which did not differ from the average of $14.7 \mathrm{cc}$ consumed by Group HCL-E $[\mathrm{t}(22)=.79$, $\mathrm{p}>.2]$. The lack of any differences in consumption as a function of extinction suggests that no stimulus generalization occurred between the flavor paired with poison and the flavor encountered on the first test trial. In other words, using a generalization of extinction paradigm counterbalanced between $\mathrm{NaCl}$ and $\mathrm{HCl}$ for training + extinction and test flavors, further evidence was obtained indicating that no significant stimulus generalization between the $\mathrm{NaCl}$ and $\mathrm{HCl}$ solutions had occurred. The present findings, especially when added to the other previously stated reasons for rejecting generalization between our salty and sour solutions, provide a compelling argument that the decreases in neophobia to $\mathrm{NaCl}$ and $\mathrm{HCl}$ seen in Experiments 1-3 following exposure to three distinctively different novel flavors were not a consequence of stimulus generalization.

\section{EXPERIMENT 5}

All groups receiving $\mathrm{LiCl}$ on Day 19 of Experiments 1-3 exhibited low acceptance of the test solution on Day 22. Despite the fact that Day 22 intake of every group in Experiments 1, 2, and 3 differed from zero, one may still suspect that the observed comparable degrees of CTA independent of prior exposure to dissimilar flavors resulted from a floor effect. To examine this possibility, Experiment 5 replicated the basic paradigm of the earlier studies, but used a weaker concentration of $\mathrm{LiCl}$. The critical differences in Experiments 1-3 were the reductions in neophobia to $\mathrm{NaCl}$ and $\mathrm{HCl}$ in the absence of any impairment in the propensity of these flavors to form CTAs; therefore, the present study was restricted to determining if the apparent absence of impairments in CTA acquisition to these two flavors may have been the result of a CTA floor effect. Moreover, given the consistent performance of the nonpoisoned groups in the previous experiments, no such controls were included in this study.

\section{Method}

Subjects and Apparatus. Twenty-four naive male rats similar in origin, maintenance, and size to those of the earlier studies were assigned randomly to one of two groups $(n=12)$ after counterbalancing for body weight. The apparatus and fluid concentrations were the same as in Experiments 1.4 except for the $.75 \%$ body-weight injection of $\mathrm{LiCl}$, which was diluted to $.15 \mathrm{M}$.

Procedure. Group DNT-CS-LiCl was exposed to $30 \mathrm{~min} /$ day of sucrose on Days 1-4, quinine on Days 7-10, $\mathrm{HCl}$ on Days 13-16, and $\mathrm{NaCl}$ on Days 19 and 22. Ten minutes after the end of the $\mathrm{NaCl}$ exposure on Day 19, this group was injected with the diluted $\mathrm{LiCl}$ solution. Hence, Group DNT-CS-LiCl was treated identically to Group DNT-CS-LiCl in Experiment 1 and Groups SucQuin- $\mathrm{HCl}$ in Experiment 3. Group $\mathrm{CS}-\mathrm{LiCl}$, composed of the remaining animals in the present experiment, received no experimental flavor exposures on Days 1-18, but the same treatment as Group DNT-CS-LiCl on Days 19 and 22.

\section{Results and Discussion}

Relative to Group CS-LiCl, Group DNT-CS-LiCl displayed greater intake of $\mathrm{NaCl}$ on Day 19 but equivalent intake of $\mathrm{NaCl}$ on Day 22 .

On Days 1-4, 7-10, and 13-16, Group DNT-CS$\mathrm{LiCl}$ displayed intakes of sucrose, quinine, and $\mathrm{HCl}$ similar to Group DNT-CS-LiCl in Experiment 1 (see Figure 1) and Group Suc-Quin- $\mathrm{HCl}$ in Experiment 3 (see Figure 3). On Day 19, animals in Group DNTCS- $\mathrm{LiCl}$ consumed an average of $26.4 \mathrm{cc}$ of $\mathrm{NaCl}$, which exceeded the $15.7 \mathrm{cc}$ mean consumption of Group CS-LiCl $[\mathrm{t}(22)=2.45, \mathrm{p}<.05]$. On Day 22 , Group DNT-CS-LiCl animals drank an average of $7.4 \mathrm{cc}$ of $\mathrm{NaCl}$, whereas mean intake of Group CS$\mathrm{LiCl}$ was $6.9 \mathrm{cc}$. These two values did not differ significantly from one another $[\mathrm{t}(22)=.19, \mathrm{p}>.5]$. However, Groups DNT-CS-LiCl and CS-LiCl both showed reductions in consumption on Day 22 relative to Day $19[\mathrm{t}(11)=3.82, \mathrm{p}<.005$, and $\mathrm{t}(11)=$ $2.22, \mathrm{p}<.05$, respectively]. Groups DNT-CS-LiCl and CS-LiCl also each differed from zero intake of Day $22[\mathrm{t}(11)=2.29, \mathrm{p}<.05$, and $\mathrm{t}(11)=2.24$, $\mathrm{p}<.05$, respectively]. Both groups displayed unusually high variance on Day 22 compared with poisoned animals in Experiments 1-3; however, the means of the present groups were so similar on Day 22 that it is not likely that the large within-group variance obscured any real difference in CTA.

The present data are consistent with the previous experiments in demonstrating that prior exposure to novel flavors dissimilar to the test substance reduces neophobia to the test substance. Moreover, the sim- 
ilarity of substantial intakes on Day 22 obviates the possibility of a floor effect and indicates that comparable CTA was induced in each group regardless of their previous treatments.

\section{GENERAL DISCUSSION}

In summary, these studies are basically consistent with, and are an extension of, the findings of Braveman and Jarvis (1978). They provide a superior basis for rejecting stimulus generalization as a significant factor in reducing neophobia by virtue of our choice of flavors and the results of Experiments 3 and 4, and they speak to the generality of the findings across flavors. Specifically, the present experiments suggest that prior exposure to diverse novel tastes maximally dissimilar to the test substance (McBurney \& Gent, 1979) reduces neophobia to $\mathrm{HCl}$ and $\mathrm{NaCl}$, but not to sucrose or quinine, and does not attenuate the potential of a flavor to form CTAs. The qualifications raised in the Discussion of Experiment 2 suggests that the above null results are possibly, although not likely, a product of the CS concentrations used; however, it is notable that reductions in neophobia to $\mathrm{NaCl}$ and $\mathrm{HCl}$ were observed at all.

Before reflecting on the implications of these findings, one further qualification concerning the generality of the observed effects is in order. The impact upon neophobia of exposure to diverse novel flavors dissimilar to the test substance is apparently contingent upon noninjurious consequences of ingesting these diverse flavors. If such exposures are followed by an internal malaise, neophobia is apparently augmented rather than attenuated (e.g., Best \& Batson, 1977).

These experiments were performed to probe the discrepancy between the observation that experience with novelty per se reduces flavor neophobia and the view that rats are subject to an exaggerated neophobia as a consequence of their varied diets, an effect perhaps augmented by the selective pressure of man's attempts to control them through the use of poisons (Barnett \& Cowan, 1976). As the wild rat, at least the urbanized one if not its country cousin, encounters a high frequency of novel flavors, it would seem that those rats that would benefit most from neophobia would have experienced the greatest attenuation of it, seemingly a maladaptive situation.

The present data do not resolve this dilemma. However, wild rats possess initially greater neophobia than our laboratory-bred subjects (Mitchell, 1976). (Note that neophobia at its greatest never attenuated intake of our albino domesticated rats below $50 \%$ of asymptotic levels, hardly a robust defense mechanism.) Consequently, the present attenuation in neophobia might prove to be relatively smaller in wild rats. Moreover, in their natural setting, wild rats would be likely to experience novel tastes with tem- poral spacings quite different from those used in the present study. Hill (1978) has pointed out that the preferences of animals for novel stimuli integrally depend upon the time between initial exposure and testing. These are but a few of the ways in which the present dilemma could be no more than a consequence of the artificiality of the laboratory situation.

An alternative interpretation of the above dilemma focuses on the possibility that neophobia did not evolve primarily as a defense against poisoning. This possibility is supported by the fact that, at present concentrations, prior exposure to novel substances decreased neophobia to $\mathrm{NaCl}$ and $\mathrm{HCl}$, but did not affect their potential to serve as CSs in CTAs. The apparent decoupling of neophobia and CTA is consistent with the observations of Braveman and Jarvis (1978) and raises questions about the relationship of neophobia to CTA, an issue first discussed in depth by Kalat and Rozin (1973) and Rozin and Kalat (1971). The present data suggest, subject to the above qualifications, that separate mechanisms underlie neophobia and conditioned taste aversions, a view consistent with the observation of Hankins, Garcia, and Rusiniak (1973) that, in rats, taste is the preferred modality for baitshyness, whereas olfaction is the preferred modality for neophobia. This possible difference in mechanisms plus the ease with which neophobia to $\mathrm{NaCl}$ and $\mathrm{HCl}$ were reduced without direct experience with these flavors, indicate, contrary to Barnett and Cowan (1976), that the primary function of neophobia may not be to minimize intake of poisons. Alternative functions of neophobia in foraging strategies should be considered. However, the possibility should not be overlooked that this apparent dilemma may be one of nature's imperfections. Natural selection averages over situations and does not yield an optimal fit to each and every ecological problem.

It is not clear whether the effects of dissimilar flavor exposure upon neophobia to $\mathrm{NaCl}$ and $\mathrm{HCl}$ differed from those upon neophobia to sucrose and quinine because a performance ceiling obscured greater initial neophobia to the latter two substances or because of a fundamental imperviousness by the latter flavors to indirect attenuation of neophobia. However, both possibilities suggest that manifest neophobias to sucrose and quinine are relatively more resistant to dilution by prior experience with dissimilar tastes. This would be consistent with the highly speculative view that sweet and bitter flavors are inherently more salient to rats as a result of the fact that sweetness often connotes high caloric food stuffs and bitterness frequently indicates toxic substances in the natural habitat of the rat. Salty and sour flavors, on the other hand, may be less highly correlated with specific outcomes of ingestion in the rat. Although salt is a necessary nutrient and domesticated rats clearly can associate salty substances with 
recovery from sodium deprivation (Rozin \& Kalat, 1971), modest amounts of salt are ordinarily present in much of the rat's natural diet.

The final point to be made is that our rats, in reducing their neophobia to $\mathrm{NaCl}$ and $\mathrm{HCl}$ as a result of prior experience with highly dissimilar flavors that minimized the possibility of stimulus generalization, behaved as if they had altered their response to novel gustatory stimuli per se. Conservative interpretations of this conclusion include the possibilities that rats have a single special mechanism regulating their response to novel stimuli (Domjan, 1976), and that exposure to novelty of any sort reduces emotional responsitivity (Braveman, 1978); however, both of these views would have to be limited to gustatory or perhaps olfactory modalities to avoid flavor neophobias' being severely attenuated by novel visual, auditory, and tactile events. Alternatively, as novelty per se has no unique stimulus properties of its own, the foregoing conclusion could be construed as suggesting that rats possess a surprisingly high potential to abstract; their altered behavior is analogous to their having formed a concept of gustatory novelty per se, to which they have then reduced their initial fear. Obviously, there is a large step between the observed attenuation of neophobia and abstraction in the traditional sense, but the potential similarity of processes is supported by other examples of concept formation in infraprimates (e.g., Morgan, Fitch, Holman, \& Lea, 1976) and suggests avenues for further research.

\section{REFERENCE NOTE}

1. Austin, T. M. Taste aversion learning: Convergent and divergent evolution. Paper presented at the meeting of the Eastern Psychological Association, Philadelphia, April 1974.

\section{REFERENCES}

BARKe R, L. M. CS duration, amount, and concentration effects in conditioned aversions. Learning and Motivation, 1976, 7, 265-273.

Barnetr, S. A., \& Cowan, P. E. Activity, exploration, curiosity and fear: An ethological study. Interdisciplinary Science Reviews, 1976, 1, 43-62.

Best, M. R. Conditioned inhibition and latent inhibition in tasteaversion learning: Clarifying the role of learned safety. Journal of Experimental Psychology: Animal Behavior Processes, 1975, 1,97-113.

Best, M. R., \& Batson, J. D. Enhancing the expression of flavor neophobia: Some effects of the ingestion-illness contingency. Journal of Experimental Psychology: Animal Behavior Processes, 1977, 3, 132-143.

Bond, N., \& DiGiusto, E. Amount of solution drunk is a factor in the establishment of taste aversion. Animal Learning \& Behavior, 1975, 3, 81-84.

Braveman, N. S. Preexposure to feeding-related stimuli reduces neophobia. Animal Learning \& Behavior, 1978, 6, 417-422.

Braveman, N. S., \& Crane, J. Amount consumed and the formation of conditioned taste aversions. Behavioral Biology, $1977,21,470-477$.

Braveman, N. S., \& Jarvis, P. S. Independence of neophobia and taste aversion learning. Animal Learning \& Behavior, 1978, 6, 406-412.

Capretta, P. J., Petersik, J. T., \& Stewaht, D. J. Acceptancy of novel flavours is increased after early experience with diverse tastes. Nature, 1975, 254, 689-691.

DAWLEY, J. M. Generalization of the CS-preexposure effect transfers to taste-aversion learning. Animal Learning \& Behavior, 1979, 7, 23-24.

DeUTSCH, R. Effects of CS amount on conditioned taste aversion at different CS-US intervals. Animal Learning \& Behavior, $1978,6,258-260$.

Domsan, M. CS preexposure in taste-aversion learning: Effects of deprivation and preexposure duration. Learning and Motivation, 1972, 3, 389-402.

Domjan, M. Poison-induced neophobia in rats: Role of stimulus generalization of conditioned taste aversions. Animal Learning \& Behavior, 1975, 3, 205-212.

Domjan, M. Determinants of the enhancement of flavored-water intake by prior exposure. Journal of Experimental Psychology: Animal Behavior Processes, 1976, 2, 17-27.

Hankins, W. G., Garcia, J., \& Rusiniak, K. W. Dissociation of odor and taste in baitshyness. Behavioral Biology, 1973, 8, 407-419.

Hennessy, M. B., Smotherman, W. P., \& Levine, S. Early olfactory enrichment enhances later consumption of novel substances. Physiology \& Behavior, 1977, 19, 481-483.

HiLl, W. F. Effects of mere exposure on preferences in nonhuman mammals. Psychological Bulletin, 1978, 85, 1177-1198.

Kalat, J. W. Should taste-aversion learning experiments control duration or volume of drinking on the training day? Animal Learning \& Behavior, 1976, 4, 96-98.

Kalat, J. W., \& Rozin, P. "Learned safety" as a mechanism in long-delay taste-aversion learning in rats. Journal of Comparative and Physiological Psychology, 1973, 83, 198-207.

Lubow, R. E., \& Moore, A. U. Latent inhibition: The effect of nonreinforced preexposure to the conditioned stimulus. Journal of Comparative and Physiological Psychology, 1959, 52, 415-419.

McBurney, D. H., \& Gent, J. F. On the nature of taste qualities. Psychological Bulletin, 1979, 86, 151-167.

Mclaurin, W. A., Farley, J. A., \& Scarborough, B. B. Inhibition effect of preirradiation saccharin habituation on conditioned avoidance behavior. Radiation Research, 1963, 18, 473-478.

Mitchell, D. Experiments on neophobia in wild and laboratory rats: A reevaluation. Journal of Comparative and Physiological Psychology, 1976, 90, 190-197.

Morgan, M. J., Fitch, M. D., Holman, J. G., \& Lea, S. E. G. Pigeons learn the concept of an "A." Perception, 1976, 5, 57-66.

Rozin, P., \& KALAT, J. W. Specific hungers and poison avoidance as adaptive specializations of learning. Psychological Review, $1971,78,459-486$.

Rzoska, J. Bait shyness, a study in rat behavior. British Journal of Animal Behaviour, 1953, 1, 128-135.

SIEGEL, S. Flavor preexposure and "learned safety." Journal of Comparative and Physiological Psychology, 1974, 87, 1073-1082.

TARPY, R. M., \& McINTosh, S. M. Generalized latent inhibition in taste-aversion learning. Bulletin of the Psychonomic Society, $1977,10,379-381$.

ZAHORIK, D. M. The role of dietary history in the effects of novelty on taste aversions. Bulletin of the Psychonomic Society, $1976,8,285-288$.

(Received for publication May 14, 1980; revision accepted November 7,1980 .) 